\title{
Evolution of flower shape in Plantago lanceolata
}

\author{
Wesley Reardon · David A. Fitzpatrick • \\ Mario A. Fares · Jacqueline M. Nugent
}

Received: 16 December 2008/Accepted: 25 June 2009/Published online: 11 July 2009

(C) Springer Science+Business Media B.V. 2009

\begin{abstract}
Plantago lanceolata produces small actinomorphic (radially symmetric), wind-pollinated flowers that have evolved from a zygomorphic, biotically pollinated ancestral state. To understand the developmental mechanisms that might underlie this change in flower shape, and associated change in pollination syndrome, we analyzed the role of CYC-like genes in P. lanceolata. Related zygomorphic species have two $C Y C$-like genes that are expressed asymmetrically in the dorsal region of young floral meristems and in developing flowers, where they affect the rate of development of dorsal petals and stamens. Plantago has a single $C Y C$-like gene $(P l C Y C)$ that is not expressed in early floral meristems and there is no apparent asymmetry in the pattern of $\mathrm{PlCYC}$ expression during later flower development. Thus, the evolution of actinomorphy in Plantago correlates with loss of dorsal-specific $C Y C$-like gene function. $P l C Y C$ is expressed in the inflorescence stem, in pedicels, and relatively late in stamen development, suggesting a novel role for $P l C Y C$ in compacting the inflorescence and retarding stamen elongation in this wind pollinated species.
\end{abstract}

Keywords Plantago - Flower shape · Protogyny · Wind pollination $\cdot$ CYCLOIDEA

W. Reardon · D. A. Fitzpatrick · J. M. Nugent $(\bowtie)$ Department of Biology, National University of Ireland, Maynooth, Co. Kildare, Ireland

e-mail: jackie.nugent@nuim.ie

M. A. Fares

Department of Genetics, Smurfit Institute of Genetics, Trinity College, Dublin, Ireland

\section{Introduction}

Flowering plants have evolved huge diversity in their floral form and in their pollination strategies. One of the most variable morphological characters is flower shape. Flowers can be classified as zygomorphic (having only one plane of reflectional symmetry or bilaterally symmetric), actinomorphic (having multiple planes of symmetry or radially symmetric) or asymmetric (having no plane of symmetry) (Endress 2001). The gene regulatory network that determines zygomorphy is best understood in the model plant Antirrhinum majus (Corley et al. 2005). In Antirrhinum zygomorphy is evident primarily in the distinct shape of the dorsal, lateral and ventral petals of the flower (Fig. 1a, Corley et al. 2005). Two genes, CYCLOIDEA (CYC) and DICHOTOMA (DICH), play a key role in promoting dorsal identity in Antirrhinum (Luo et al. 1996, 1999). CYC and $\mathrm{DICH}$ are paralogous genes (CYC-like genes); both are expressed throughout floral development in dorsal regions and encode proteins belonging to the TCP family of transcription factors (Cubas et al. 1999a). In addition to affecting petal shape, $C Y C$-like gene activity in dorsal regions inhibits stamen growth in Antirrhinum and the dorsal stamen is reduced to a staminode (Luo et al. 1996; Luo et al. 1999). In Antirrhinum cyc/dich double mutant plants produce flowers that are actinomorphic, with all petals and stamens resembling the ventral petals and stamens of wild-type flowers (Carpenter and Coen 1990).

Zygomorphic flowers have evolved independently from actinomorphic ancestors many times throughout flowering plant evolution (Stebbins 1974; Donoghue et al. 1998; Endress 2001). CYC-like genes have been co-opted into at least two separate genetic programs promoting floral asymmetry in angiosperms, once within the asterids (Antirrhinum and relatives), and once within the rosids 
(a)

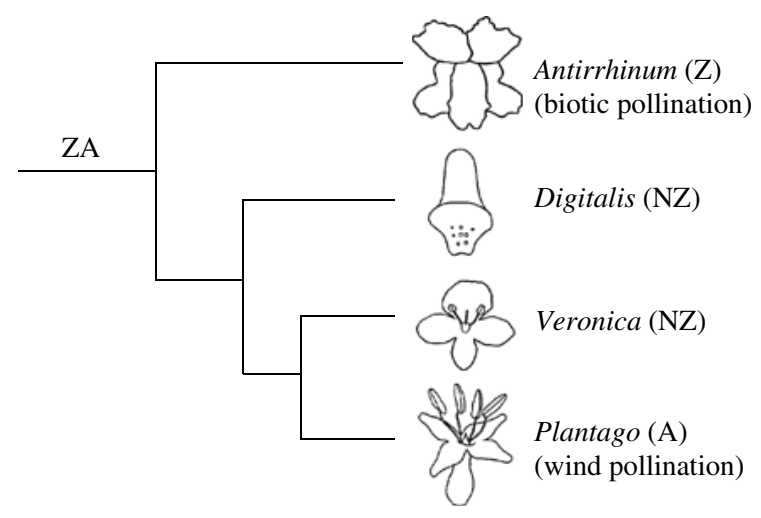

(b)

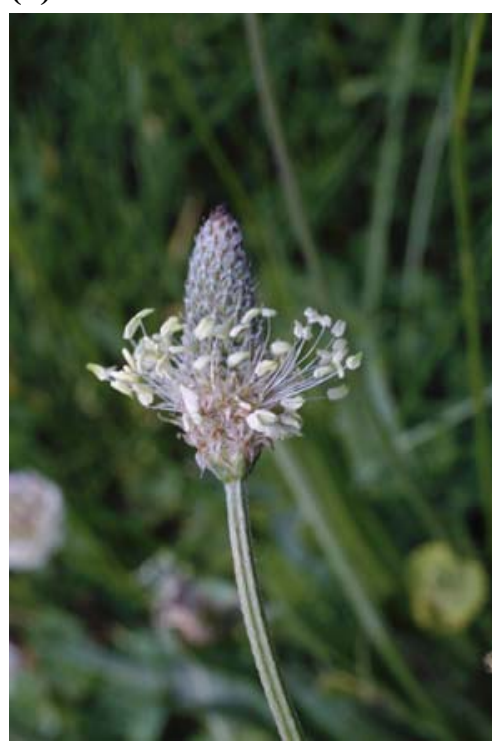

Fig. 1 P. lanceolata flowers are actinomorphic and wind pollinated and are derived from a zygomorphic and biotic pollinated ancestral state. a Schematic representation of the progression from zygomorphy $(\mathrm{Z})$, and biotic pollination, to actinomorphy (A), and wind pollination, within the Veronicaceae (only some representative genera are shown and flowers are not drawn to scale). The ancestor of this clade is

(Lotus and other legumes) (Cubas 2004). CYC-like genes are expressed in a generalized dorsoventral pre-pattern in meristems involved in both flower and shoot formation, even in species with actinomorphic flowers, suggesting that the origin of the genes predates their role in zygomorphy (Cubas et al. 2001; Clark and Coen 2002). However, it is only in zygomorphic species that $C Y C$-like gene expression is maintained during flower development in dorsal floral organs (Luo et al. 1996; Luo et al. 1999; Cubas et al. 1999b; Hileman et al. 2003; Feng et al. 2006; Citerne et al. 2006; Wang et al. 2008). This acquisition and maintenance of $C Y C$ expression in dorsal floral organs may have been the driving force behind the evolution of dorsoventral floral asymmetry (Clark and Coen 2002). Changes in the regulation of $C Y C$-like genes have also played a causal role in the evolution of derived zygomorphic variants within the Antirrhineae. Mohavea confertiflora has zygomorphic flowers with three stamens aborted (one dorsal and two lateral), and a corolla that is more radially symmetrical than the corolla of the Antirrhinum flower. In Mohavea, an expansion of the domain of expression of $C Y C$-like genes during flower development correlates with this derived floral morphology (Hileman et al. 2003). CYC-like genes have also played a role in the evolution of flower shape in legumes (Feng et al. 2006; Citerne et al. 2006; Wang et al. 2008). In the zygomorphic papilinonoid legume Lupinus nanus, a $C Y C$-like gene is expressed in dorsal floral regions in a pattern similar to Antirrhinum (Citerne et al. 2006). inferred to have had zygomorphic flowers $(\mathrm{ZA}=$ zygomorphic ancestor; $\mathrm{NZ}=$ near zygomorphy); $\mathbf{b} P$. lanceolata produces a compact inflorescence of small, actinomorphic, wind-pollinated flowers subtended by a leafless scape. Stamen exsertion and floral anthesis proceeds from the bottom to the top of the inflorescence after female flowering (image courtesy of Steven J. Baskauf)

However, in the closely related but actinomorphic, species Cadia purpurea, CYC-like gene expression occurs in all floral regions (Citerne et al. 2006). Thus, in Cadia and Mohavea, loss of zygomorphy is not achieved by a loss of function event, but by a regulatory event that results in an expansion of the domain of $\mathrm{CYC}$-like gene expression during flower development. $C Y C$-like genes also affect the rate of stamen development and eventual stamen size in legumes (Citerne et al. 2006).

Zygomorphic flowers predominate within the Lamiales, although the basal lineages produce actinomorphic flowers (Endress 1997; Olmstead et al. 2000; Bremer et al. 2002). Molecular systematic studies of species within the Lamiales place Antirrhinum within the Veronicaceae, a very highly supported clade that includes the Antirrhineae, Digitaleae and Plantaginaceae (Olmstead et al. 2001). Within this clade there is a progression from pronounced zygomorphy in Antirrhinum to reduced zygomorphy in Digitalis and Veronica, to actinomorphy in Plantago, accompanied by a shift from biotic to wind pollination (Fig. 1a). Wind pollination (anemophily) is predominantly a derived condition in flowering plants, and has evolved at least 65 times from biotically pollinated ancestors (Friedman and Barrett 2008). Wind pollinated plants tend to share several morphological traits that constitute the "anemophilous syndrome" including small unisexual or dichogamous flowers (have temporal separation of male and female organs within a flower) and condensed inflorescences borne on elongate subtending 
internodes (Culley et al. 2002; Weller et al. 2006; Friedman and Barrett 2008). Two forms of dichogamy exist: protandry (male phase precedes female) and protogyny (female phase precedes male). Protandry is generally associated with biotic pollination and protogyny is generally associated with wind pollination (Bertin and Newman 1993). Phylogenetic analysis points to a possible link between floral symmetry and dichogamy: within the Asteridae protandry has been lost more frequently than expected in actinomorphic clades and has been gained more frequently than expected in zygomorphic clades. This suggests that zygomorphy and protandry may be correlated and potentially controlled by the same regulatory genes (Kalisz et al. 2006).

The phylogenetic placement of Plantago within the Veronicaceae makes it an excellent system in which to investigate the developmental mechanisms that might underlie reversals to actinomorphy and associated changes in pollination syndrome. Plantago lanceolata produces a basal rosette of lanceolate leaves during vegetative growth and at flowering produces several leafless scapes, each bearing a compact inflorescence of tiny, actinomporphic, flowers (Fig. 1b). The flowers are arranged in a spiral phylotaxy, and are subtended by bracts. Flowers consist of four sepals, four petals, four stamens, and a single pistil of two fused carpels (Henderson 1926). One of the most distinctive features of flowering in Plantago lanceolata is that the flowers are protogynous, i.e., the stigmas are exserted and become receptive before the anthers are mature. When female flowering is nearly complete, the flowers at the base of the inflorescence open, exsert their stamens, and release pollen that is wind dispersed. Male flowering then proceeds up the inflorescence (Fig 1b). The question addressed in this study is whether the relatively recent shift to actinomorphy, and concomitant shift to protogyny and wind pollination, in Plantago might have a common genetic cause involving flower shape genes. To this end, we characterized $C Y C$-like genes in Plantago lanceolata.

\section{Results}

\section{CYC-like genes in Plantago lanceolata}

All of the PCR reactions carried out on Plantago genomic DNA and cDNA generated a single size class of PCR product for each primer pair used. Control PCR carried out on Antirrhinum genomic DNA and cDNA, using the same primer pair combinations, generated the two expected size classes of PCR products (CYC and DICH, data not shown). In total, 19 Plantago cloned PCR products were sequenced (eight $\mathrm{CycP} 1 / \mathrm{CycP} 2$ products; four TCP1F/CycP2 products; seven $\mathrm{Cyc} 2 \mathrm{~F} / \mathrm{Cyc} 3 \mathrm{R}$ products). Percent divergences (uncorrected $p$-distances) between all Plantago lanceolata sequences is $<1.1 \%$. This is within the range of sequence divergence rates reported for other $C Y C$-like alleles in the Veronicaceae (0.53-1.42\%, Hileman and Baum 2003) suggesting that all Plantago sequences represent alleles of a single $C Y C$-like gene locus (PlCYC). Phylogenetic analyses were carried out with one of the Plantago sequences and $C Y C$-like gene sequences from other Veronicaceae to assess gene orthology. Maximum likelihood and Bayesian methods yielded a single tree (Fig. 2). The Plantago sequence emerges as sister group to the Digitalis $C Y C 2$ and $C Y C 3$ genes that have arisen as a result of a recent gene duplication event within the Digitalis lineage (Hileman and Baum 2003). Both the ML and Baysian trees suggest a $C Y C$-like gene duplication event occurred before the radiation of the Antirrhineae, giving

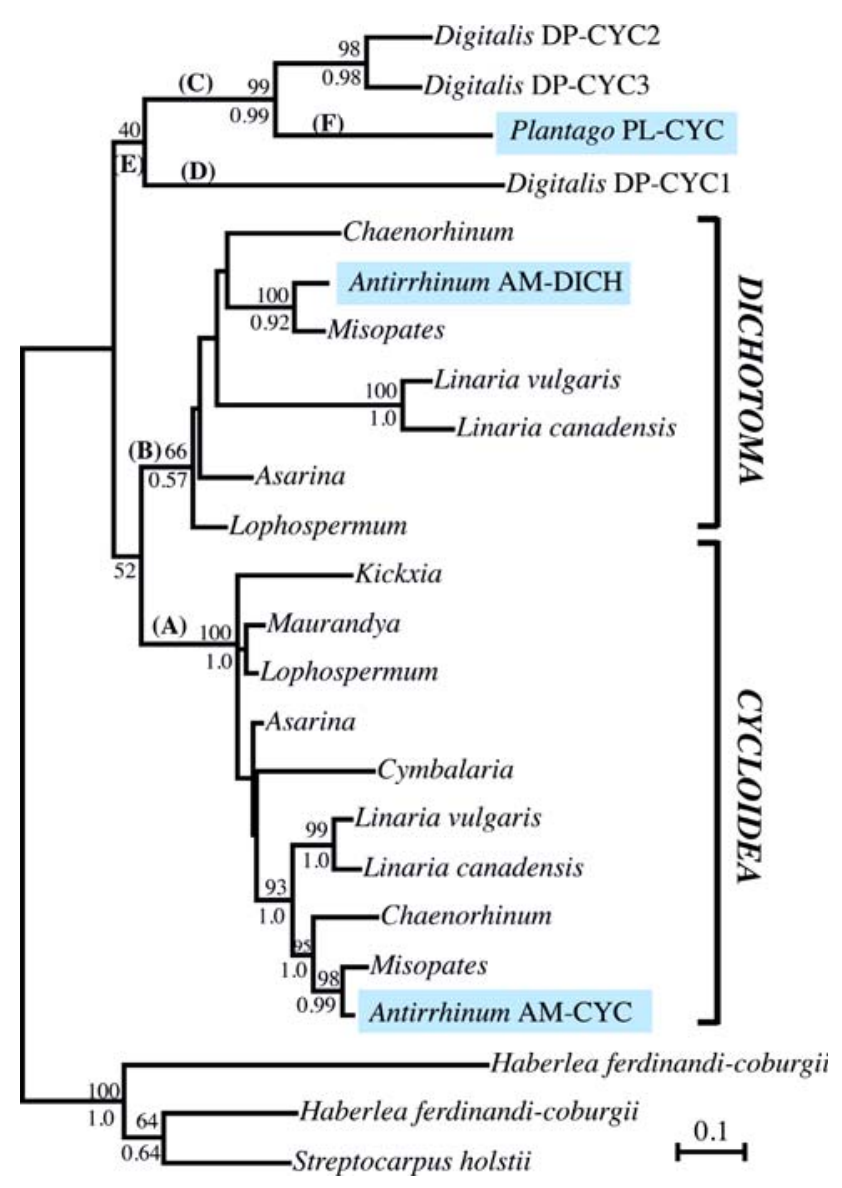

Fig. 2 Maximum likelihood phylogeny representing the relationships among $C Y C$-like loci of the Antirrhineae and close relatives. The optimum model of protein substitution was found to be JTT + G. The number of gamma rate categories was $4(\alpha=0.71)$. Bootstrap re-sampling (100 iterations) was undertaken and values are displayed above selected branches. Bayesian posterior probabilities are shown below selected branches. Letters (A-F) in parentheses are used to distinguish branches that were tested for varying $\omega$ values. The Haberlea and Streptocarpus (Gesneriaceae) CYC-like sequences are included as an outgroup 
(1)

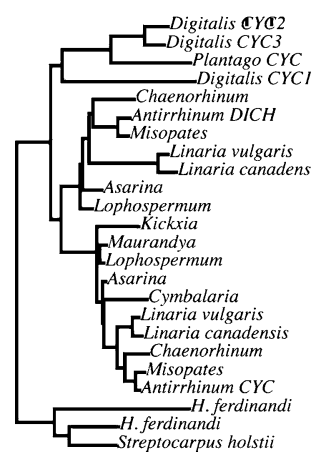

$-6184.246768$

(6)

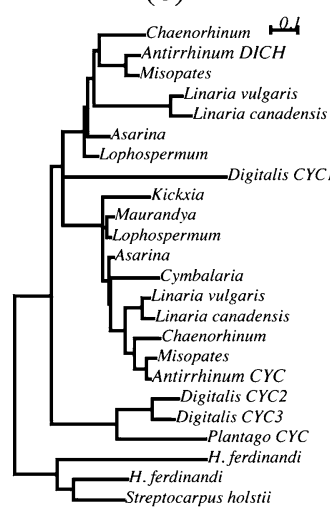

$-6189.836874$
(2)

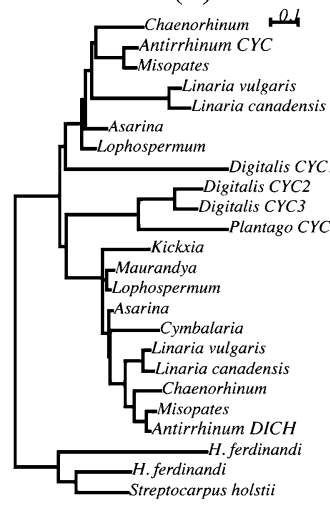

$-6188.418830$
(3)

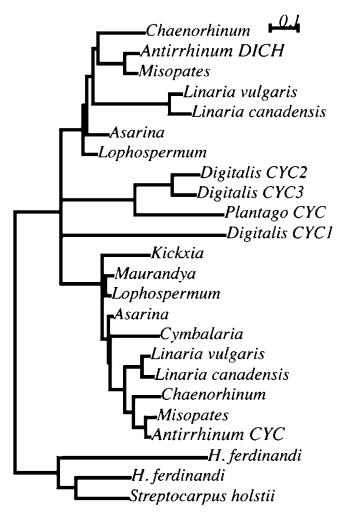

$-6190.387644$
(4)

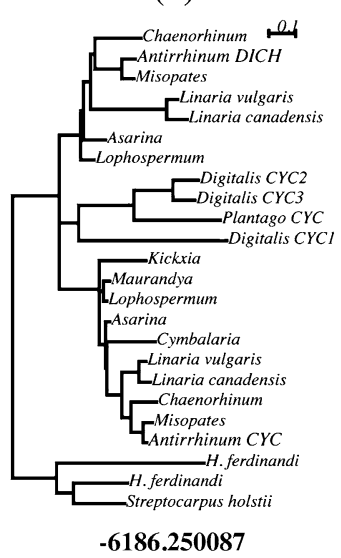

(5)

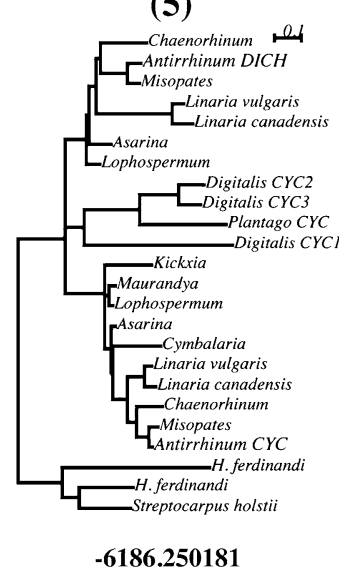

(8)

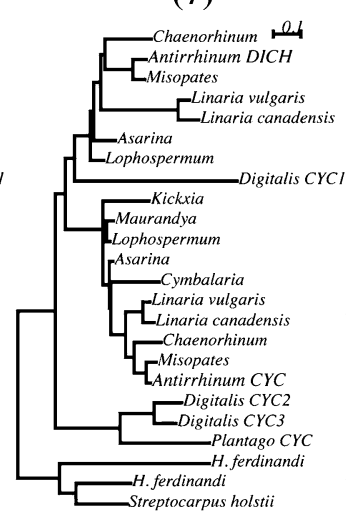

$-6188.177863$

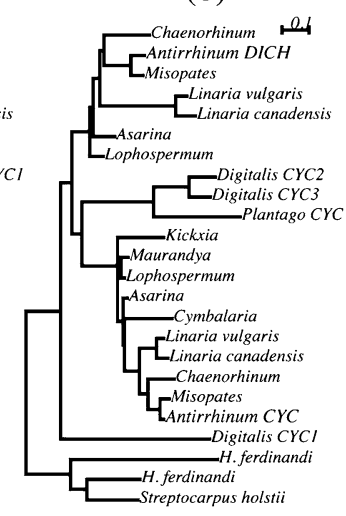

$-6188.564916$
(9)

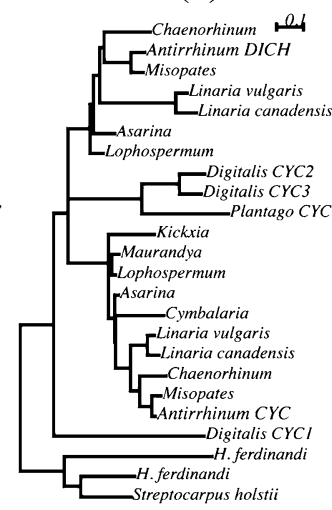

$-6189.949808$
(10)

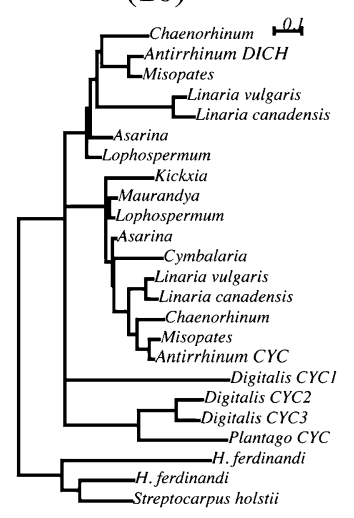

$-6190.386575$
Fig. 3 Constrained and unconstrained trees used for the approximately unbiased test. Tree 1 is the original unconstrained topology (Fig. 2). Topologies 2-10 are constrained topologies that infer different branching scenarios for $C Y C, D I C H$ and the Plantago and Digitalis $C Y C$-like gene sequences. Log likelihood scores are given

rise to $C Y C$ and $D I C H$ clades (Fig. 2 clades A and B). However, bootstrap support for the $C Y C / D I C H$ clade is very low (52\%), and likewise for the Digitalis/Plantago clade (40\%). Therefore, we tested alternative relationships by using the approximately unbiased test of phylogenetic tree selection to assess if differences in topology between constrained and unconstrained gene trees are greater than expected by chance. Nine different constrained topologies were assessed (Fig. 3), and although the unconstrained tree received the optimal likelihood tree score, this was not significantly different from the constrained trees $(P>0.05)$. Based on these results, the placement of $C Y C$ and $D I C H$ sequences, to the exclusion of the Digitalis and Plantago sequences is not significant. Thus, we cannot rule out the possibility that the $C Y C / D I C H$ gene duplication event occurred before the divergence of the lineage that gave rise to Plantago and Digitalis from the Antirrhineae lineage. Such an early duplication in the evolution of the Veronicaceae would suggest that a $C Y C$-like gene was lost below each tree. The approximately unbiased (AU) test was used to assess likelihood scores for each topology and the differences in likelihood scores between the original tree and constrained trees are not significant $(P>0.05)$

during Plantago evolution. We used a maximum likelihood (ML) approach to assess the selective constraints that have driven the evolution of $\mathrm{CYC}$-like genes in the Veronicaceae. A likelihood ratio test (LRT) was used to look for evidence of variation in the rate of nonsynonymous versus synonymous substitution rates $\left(d_{\mathrm{N}} / d_{\mathrm{S}}=\omega\right)$ both between sites and along different lineages. Both types of $\mathrm{ML}$ analyses provided strong evidence only for purifying selection $(\omega<1$, Tables 1,2$)$. Furthermore, LRTs indicate that models that allow for positively selected sites do not significantly increase the likelihood scores when compared with models that do not permit positive selection (Table 1). Codon-based likelihood models that allow for different $d_{\mathrm{N}} /$ $d_{\mathrm{S}}$ ratios among evolutionary lineages were also used to determine if specific lineages in the dataset are undergoing positive selection (Fig. 2, lineages A-F). None of the LRTs allowed for positive selection in any lineage, and overall $\omega$ values were found to be $<0.5$ (Table 2). These results suggest that all $\mathrm{CYC}$-like genes used in these analyses are 
Table 1 Variation in the underlying nonsynonymous/synonymous substitution rate ratio $(\omega)$ among sites

\begin{tabular}{|c|c|c|c|c|}
\hline Model & Estimates of parameters & $\operatorname{Ln} L$ & $2 \Delta \ln L$ & $\begin{array}{l}\text { Positively selected } \\
\text { codons }\end{array}$ \\
\hline M0 (one ratio) & $\omega_{1}=0.22664$ & $-11,557.50$ & & None \\
\hline M1 (neutral) & $p_{1}=0.671 p_{2}=0.328 \omega_{1}=0.125 \omega_{2}=1$ & $-11,351.98$ & & Not allowed \\
\hline M2 (selection) & $\begin{array}{l}p_{1}=0.671 p_{2}=0.198 p_{3}=0.130 \omega_{1}=0.125 \omega_{2}=1 \\
\quad \omega_{3}=1\end{array}$ & $-11,351.98$ & (M1 vs. M2) $P<1$ & None \\
\hline $\begin{array}{l}\text { M3 (discrete } \\
\quad k=2 \text { ) }\end{array}$ & $p_{1}=0.518 p_{2}=0.481 \omega_{1}=0.062 \omega_{2}=0.506$ & $-11,296.95$ & (M0 vs. M3) $P<0.0005$ & None \\
\hline M7 (beta) & $p=0.592 q=1.426$ & $-11,281.44$ & (M7 vs. M8) $P<1$ & Not allowed \\
\hline M8 (beta \& $\omega$ ) & $p=0.572 q=1.426 p_{1}=0 \omega_{1}=1$ & $-11,281.44$ & & None \\
\hline
\end{tabular}

Note: $p_{1}, p_{2}$, and $p_{3}$ refer to the proportion of sites in categories 1,2 , and 3 , respectively. $\omega$ refers to the $d_{\mathrm{N}} / d_{\mathrm{S}}$ ratio in each of the three category of sites. $p$ and $q$ are $\beta$ estimates. Models M1 and M7 do not allow positively selected sites. The number of degrees of freedom for all likelihood ratio tests is 2

Table 2 Variation in the underlying nonsynonymous/synonymous substitution rate ratio $(\omega)$ among selected lineages

\begin{tabular}{lllll}
\hline Model & $\omega_{1}$ & $\omega_{2}$ & $\ln L$ & $2 \Delta \ln L$ \\
\hline 1 ratio & $\omega_{1}=0.2266$ & N/A & $-11,557.504870$ & \\
2 ratio-A & $\omega_{1}=0.2233$ & $\omega_{2}=0.3654$ & $-11,556.798376$ & $P<0.9$ \\
2 ratio-B & $\omega_{1}=0.2243$ & $\omega_{2}=0.3092$ & $-11,557.146465$ & $P<0.9$ \\
2 ratio-C & $\omega_{1}=0.2233$ & $\omega_{2}=0.2520$ & $-11,556.266664$ & $P<0.9$ \\
2 ratio-D & $\omega_{1}=0.2240$ & $\omega_{2}=0.3710$ & $-11,556.896408$ & $P<0.9$ \\
2 ratio-E & $\omega_{1}=0.2219$ & $\omega_{2}=0.4215$ & $-11,555.480389$ & $P<0.9$ \\
2 ratio-F & $\omega_{1}=0.2242$ & $\omega_{2}=0.2753$ & $-11,557.126899$ & $P<0.9$ \\
Free ratio & Multiple $\omega$ all $<1$ & $-11,521.323637$ & $P<0.005$ \\
\hline
\end{tabular}

undergoing strong purifying selection, and that gene function in all lineages is likely conserved.

\section{Expression analysis of PlCYC}

RNA in situ hybridization was carried out to establish the pattern of expression of $\mathrm{PlCYC}$ during flower development (Fig. 4). No PlCYC transcripts were detected in early floral meristems (Fig. 4a). However, PlCYC is expressed within the ground tissue of the inflorescence stem, and at the base of young floral meristems in tissue that gives rise to pedicels (short stems that link the flower to the inflorescence axis; Fig. $4 \mathrm{~b}, \mathrm{c})$. As the pedicels and flowers develop, PlCYC is transiently expressed in the ground tissue of the pedicels and at the base of all four floral organs (Fig. 4d). Later in flower development (mid-stage flowers), $P l C Y C$ is expressed in all four stamens, in particular in the connective tissue of the anthers and the upper parts of the filaments (Fig. $4 \mathrm{e}-\mathrm{g}$ ). Some transient $P l C Y C$ expression is also seen within the ovaries (Fig. 4e). No PlCYC expression is detected in petals. No hybridization signal was detected in similar stage tissue sections probed with the same amount of sense control probe (data not shown). RT-PCR was also used to assess PlCYC expression in petals and stamens in mid-stage, $\mathrm{mid} /$ late-stage, and late-stage flowers. Since short stamen filaments are fused to the base of the petals, it was not possible to obtain samples of pure stamen tissue from the mid-stage flowers. However, since $P l C Y C$ expression was not detected in petals, transcripts detected in these flower tissues are likely to come from stamen tissue only (Fig. 4 and data not shown). The RT-PCR results show that $P l C Y C$ is expressed in midstage flowers, in mid/late-stage flowers when the stamens are elongated but not exserted, and continues to be expressed in late-stage flowers that have the stamens exserted (Fig. 5). RT-PCR was also used to assess PlCYC expression in the inflorescence. $P l C Y C$ is expressed throughout the inflorescence stem, prior to and after stamen exsertion and flower anthesis, but was not detected in the long subtending internode of the scape that subtends the flowers (Fig. 5).

\section{Discussion}

This study shows that the actinomorphic species Plantago lanceolata has only one $C Y C$-like gene, in contrast to most other zygomorphic relatives that contain at least two (Hileman and Baum 2003). This result is in agreement with previously published work that reported a single $C Y C$-like locus in Plantago major (Reeves and Olmstead 2003), and phylogenetic analyses show that the two Plantago sequences group together as expected (data not shown). The spatial pattern of $P l C Y C$ expression in $P$. lanceolata is strikingly different compared to $C Y C$-like gene expression in related, zygomorphic, Antirrhineae species. $P l C Y C$ is not expressed in young floral meristems, is not expressed in petals, and there is no apparent asymmetry in the pattern of PlCYC expression at any stage during flower development. Thus, actinomorphy in Plantago correlates with loss of CYC-like gene expression in early floral meristems, and in developing petal primordia. This contrasts with the 
Fig. 4 In situ hybridization expression pattern of $\mathrm{PlCYC}$ during inflorescence and flower development in $P$. lanceolata. A-D, PlCYC antisense probe hybridized to transverse sections through the $P$. lanceolata inflorescence apex (ia) and developing floral meristems and flowers. PlCYC expression is not detected in young floral meristems (fm; A) but is detected in the ground tissue of the inflorescence stem (is; B and C) and in tissue at the base of floral mounds that give rise to pedicels $(\mathrm{p} ; \mathrm{C})$. As the pedicels and flowers develop $P l C Y C$ is expressed in the ground tissue of the pedicels and at the base of all floral organs (D). E-G, PlCYC antisense probe hybridized to sections through developing $P$. lanceolata flowers. Later in flower development, PlCYC is expressed in stamens (s), in particular in anther connective tissue (ct, arrows in E and F) and in the upper part of stamen filaments (sf; G). Some transient PlCYC expression is seen within the ovaries $(\mathrm{o} ; \mathrm{E}-\mathrm{G})$. There is no apparent asymmetry in the pattern of PlCYC expression during flower development in P. lanceolata

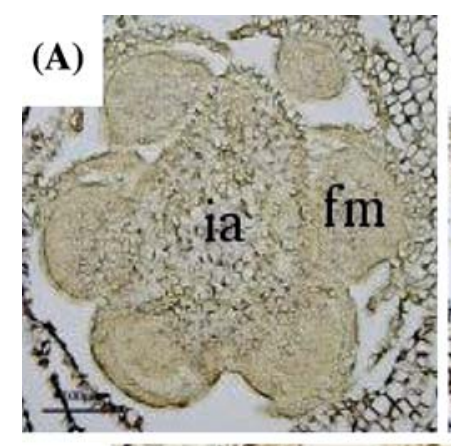

(B)

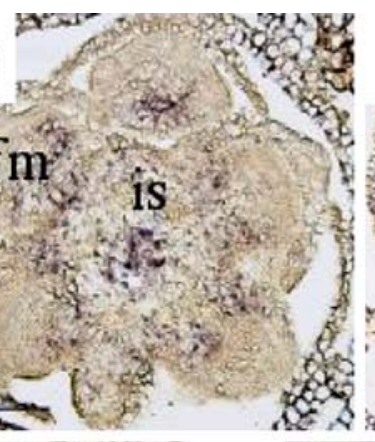

(C)

(D)
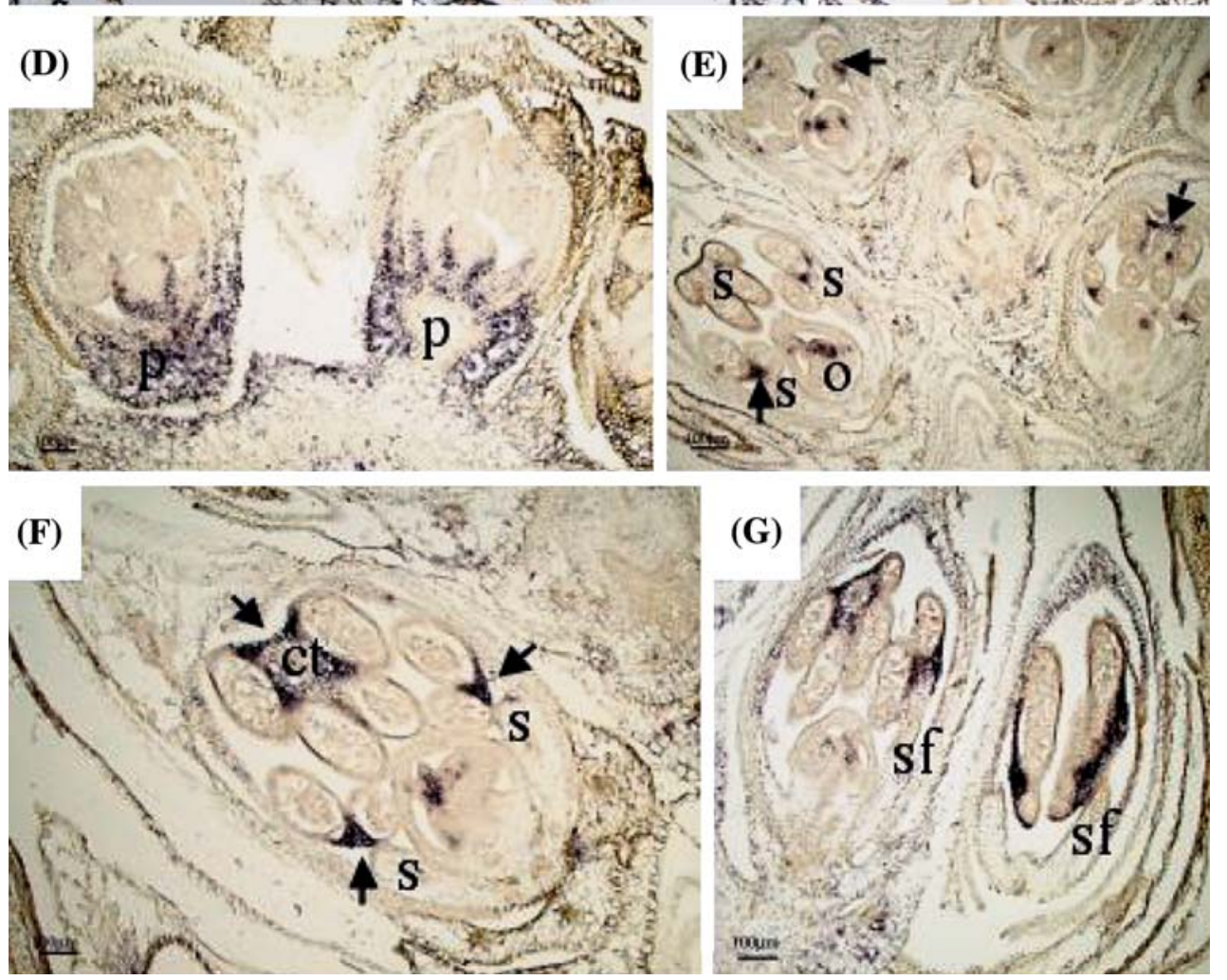

situation in Cadia and Mohavea, where loss of floral asymmetry is achieved by an expansion in the domain of CYC-like gene expression during flower development (Hileman et al. 2003, Citerne et al. 2006). In Antirrhinum, loss of $C Y C$-like gene function leads to a change in flower shape, and a concomitant increase in petal number from five to six (Luo et al. 1996). It has been argued that Plantago flower morphology does not support a loss of gene function event in the evolution of actinomorphy because Plantago flowers have four petals rather than six, i.e., a reduced rather than an increased petal number relative to Antirrhinum (Donoghue et al. 1998). However, the role of $C Y C$-like genes in determining flower shape is not always coupled to a role in determining floral organ number: loss of $C Y C$-like gene activity in Linaria vulgaris, a species very closely related to Antirrhinum, also results in actinomorphy, but with no affect on floral organ number (Cubas et al. 1999b). In addition, although pentamery is often correlated with zygomorphy, a reduction from pentamerous to tetramerous corollas has occurred at least twice within the Lamiales without loss of zygomorphy (Bello et al. 2004). This indicates that the shift to a tetramerous corolla may also be independent of $C Y C$-like gene activity. Scanning electron micrographs of early flower development in Plantago show slightly slower rates of development of petal and stamen primordia in dorsal compared to ventral positions, indicating a degree of zygomorphy that is not seen in mature flowers (Endress 1997, Bello et al. 2004). It is unclear what role, if any, PlCYC has in establishing this early zygomorphic pattern of organ development because in situ hybridization does not detect PlCYC expression during floral organ initiation in Plantago. It may be that the quite subtle differences in the rate of growth of dorsal and ventral floral organs in Plantago is mediated by such a low level of $P l C Y C$ expression that it is not detectable by in situ hybridization. In the zygomorphic Brassicaceae 


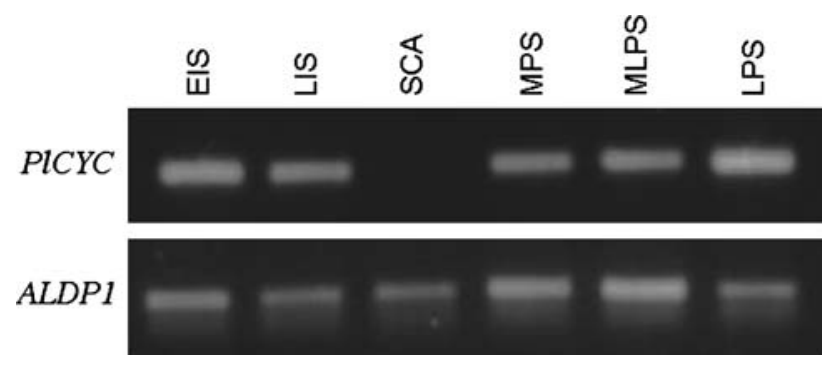

Fig. 5 Agarose gel image showing the results of RT-PCR analysis of $P l C Y C$ expression in $P$. lanceolata inflorescence tissue and floral organs. PlCYC is expressed in early inflorescence stems (EIS) and late inflorescence stems (LIS) but not in the subtending scape that bears the inflorescence (SCA). PlCYC is expressed in petal and stamen tissue isolated from mid-stage (MPS), mid/late-stage (MLPS) and late-stage (LPS) flowers. $A L D P 1$ is included as a control

species Iberis amara, relative differences in dorsal and ventral petal growth start to become apparent at the onset of stamen differentiation. However, in situ hybridization shows no obvious difference in the level of IaTCP1 expression in dorsal or ventral petal primordia at this stage (Busch and Zachgo 2007). Again, this lack of difference may be a reflection of the limitations of in situ hybridization to detect, or discriminate between, very low levels of gene expression in plant tissues. Alternatively, subtle differences in the rate of floral organ growth in dorsal and ventral regions early in flower development, both in Plantago and Iberis, may be mediated by factors other than $C Y C$-like gene activity.

Gene loss is rarely considered as a mediator of developmental change, which is more often associated with regulatory or functional differences in orthologue activity. We found no evidence for positive Darwinian selection in the $P l C Y C$ protein-coding region, as might be expected if the protein had acquired a novel function. Thus, the TCP transcription factor encoded by $\mathrm{PlCYC}$ most likely functions like other related $C Y C$-like transcription factors to regulate plant cell proliferation and growth (Gaudin et al. 2000; Corley et al. 2005; Busch and Zachgo 2007). In Plantago, it is equally plausible that $C Y C$-like gene function was lost in dorsal floral regions because of a gene loss event or a gene regulatory event. Phylogenetic analyses of CYC-like sequences indicate that a gene duplication event occurred before the divergence of the Antirrhineae, and gave rise to $C Y C$ and $D I C H$ loci (Fig. 2; Hileman and Baum 2003). However, the data cannot rule out the possibility that the gene duplication event occurred before the divergence of the Digitalis and Plantago lineages from the lineage that gave rise to the Antirrhineae (Fig. 3; Hileman and Baum 2003). Depending on the timing of the gene duplication event, two different scenarios may be presented to explain the evolution of actinomorphy in Plantago. A gene duplication event early in the evolution of the Veronicaceae suggests that a $C Y C$-like gene was lost within the Plantago lineage, and that actinomorphy in Plantago may be correlated with this gene loss event. A corollary of this scenario is that one of the duplicate genes in Plantago acquired adaptive mutations in cis-regulatory regions prior to this gene loss event. A gene duplication event later in the evolution of the Veronicaceae, after the divergence of the Digitalis and Plantago lineages but before the radiation of the Antirrhineae, suggests that evolution of actinomorphy in Plantago has involved adaptive mutations in the $c i$-regulatory regions of a single $C Y C$-like gene that previously had a role in establishing zygomorphy. Additional work will be required to resolve the timing of this gene duplication event and to discriminate between these two possible evolutionary routes to actinomorphy in Plantago.

$P l C Y C$ is expressed in the inflorescence stem and pedicels and is expressed late in stamen development in connective and filament tissue. This suggests gain of a novel developmental role for PlCYC in inflorescence compression and protogyny. $\mathrm{CYC}$-like genes are not expressed in inflorescence tissue in related Antirrhineae species. However, in maize the $C Y C$-like gene $T B 1$ affects internode length in the inflorescence, as well as suppressing axillary bud growth (Doebley et al. 1997). Constitutive expression of $T B 1$ in wheat has the generalized effect of reducing internode length in transgenic plants, and affects inflorescence architecture by reducing internode length on mature spikes, giving rise to more compact inflorescences than in wild-type plants (Lewis et al. 2008). In addition, constitutive expression of IaTCP1 in Arabidopsis resulted in some transgenic plants that were dwarfed in all aspects of their growth (Busch and Zachgo 2007). Thus, the extremely compact architecture of the Plantago inflorescence may be determined by $P l C Y C$ activity inhibiting internode and pedicel elongation in the inflorescence. In contrast, RT-PCR showed no PlCYC expression in the extremely elongated internode of the scape subtending the flowers.

Antirrhinum cyc mutants produce flowers with all stamens developing to full length, suggesting that $C Y C$ inhibits dorsal stamen growth (Luo et al. 1996). In Antirrhinum, expression of $C Y C$ in early dorsal stamen primordia negatively affects cell-cycle genes such as CYCLIN D $3 B$ (Gaudin et al. 2000). In Mohavea, an expansion of the domain of expression of $C Y C$-like genes in the stamen whorl, relative to Antirrhinum, causes abortion of both the dorsal and lateral stamens (Hileman et al. 2003). In Maize, TB1 is strongly expressed in the early stamen primordia of female ear florets, where selective stamen abortion occurs during development (Hubbard et al. 2002). These results suggest that expression of $C Y C$-like genes early in stamen development leads to stamen developmental arrest mediated by the inhibition of cell cycle genes. Iberis amara flowers are zygomorphic, and have two small adaxial and two large abaxial petals (Busch and Zachgo 2007). Expression of IaTCP1 is stronger in 
developing adaxial petals than in abaxial petals, and IaTCP 1 expression inhibits adaxial petal growth by negatively regulating cell proliferation (Busch and Zachgo 2007). In Plantago, PlCYC is expressed relatively late in stamen development, after anthers and filaments have formed but before filament elongation, and expression is localised to the connective tissue of anthers and the upper parts of the filaments in all four stamens. In situ hybridization and RT-PCR data show that $P l C Y C$ is expressed in stamens up to the stage when the stamen filaments are completely elongated and exserted. These results suggest that late expression of PICYC in Plantago may act to delay stamen filament elongation, thus achieving temporal separation of male and female flowering (dichogamy). Detailed analysis of stamen growth in Plantago lanceolata will be needed to determine if PlCYC affects cell proliferation, or indeed cell expansion, during stamen development.

The results of this study suggest that actinomorphy and protogyny may be correlated in Plantago through altered activity of $C Y C$-like genes. Macroevolutionary evidence suggests that zygomorphy and protandry may be linked via the selective action imposed by pollinator behaviour (Kalisz et al. 2006). Zygomorphic asterids are pollinated mainly by bees that rely on visual floral cues to guide them towards the flower, and a shift from zygomorphy to actinomorphy could be disadvantageous particularly when mature anthers remain within a tubular flower (Donoghue et al. 1998). However, the evolution of a genetic framework linking both flower shape and dichogamy could be one solution to achieving reproductive success, irrespective of floral shape (at least within the Veronicaceae), and may help to explain why changes in flower shape have evolved so frequently during flowering plant evolution.

Experimental procedures

\section{Plant material}

$P$. lanceolata plants grown from seed (CMS 2 type seed obtained from Jos Van Damme, Holland), and P. lanceolata plants growing wild at NUIM, Ireland were used for the gene cloning experiments. In situ hybridization experiments were carried out on inflorescence tissue obtained from $P$. lanceolata plants growing wild at the University of Edinburgh, Scotland. The tissues used for the RT-PCR experiments were obtained from $P$. lanceolata plants growing wild at NUIM, Ireland.

\section{CYC-like genes and phylogenetic analyses}

Genomic DNA and cDNA was isolated from $P$. lanceolata vegetative and inflorescence tissues, respectively, and PCR was carried out using three different primer combinations
(CycP1-5' TTGGGAAGAAGAACACATACCTA $3^{\prime} /$ Cyc P2-5' AATTGATGAACTTGTGCTGAT $3^{\prime}$; TCP1F-5' GCATTGCGAGGAAGTTCT $3^{\prime} / \mathrm{CycP} 2$; and $\mathrm{Cyc} 2 \mathrm{~F}-5^{\prime}$ AAAGATCGCCACAGCAA $3^{\prime} / \mathrm{Cyc} 3 \mathrm{R}-5^{\prime}$ TCCCTAGCT CTCGCTCTCGCCTTCGCCCT $3^{\prime}$ ). The primer pair CycP1/ $\mathrm{CycP} 2$ was previously used to amplify $C Y C$-like genes from a range of Antirrhineae and related species (Vieira et al. 1999, Hileman and Baum 2003). The TCP1F and $\mathrm{Cyc} 3 \mathrm{R}$ and $\mathrm{Cyc} 2 \mathrm{~F}$ primers were designed against conserved regions within the TCP and R domains that are characteristic of CYC-like genes (Cubas et al. 1999a). PCR products were cloned into the pCR 2.1 cloning vector and sequenced. Percent divergences (uncorrected $p$-distances) between Plantago lanceolata sequences were assessed with PAUP* ver 4.0b10 (Swofford 1998) (nucleotide differences not shared by at least two independently derived sequences were ignored as potential PCR or sequencing errors). One of the Plantago sequences (Acc. No. GQ303572) was selected and aligned with other Veronicaceae CYC-like gene sequences from GenBank (Acc. Nos: Y16313, AF199465, AF512598, AF512592, AF512601, AF512591, AF512599, AF512595, AF512602, AF512589, AF512603, AF512590, AF512596, AF512593, AF512597, AF512600, AF512594, AF512604, AF512605, AF512606, AF208322, AF208317, AF208334; Gesnericaceae CYC-like gene sequences were included as an outgroup). Deduced amino acid sequences were aligned using T-Coffee and MAFFT (Notredame et al. 2000, Katoh et al. 2005) and manually adjusted with Se-Al 2.0. Phylogenetic relationships amongst amino acid sequences were inferred with maximum likelihood methods. The appropriate protein model of substitution was determined with ModelGenerator (Keane et al. 2006). One hundred bootstrap replicates were carried out with the appropriate protein model using PHYML (Guindon and Gascuel 2003), and summarized using the majority-rule consensus method. A Bayesian phylogeny using the heterogeneous CAT site model was also reconstructed. The CAT model can account for site-specific features of sequence evolution, and has been found to be more robust than other methods against phylogenetic artifacts such as long-branch attraction (Lartillot et al. 2007). We performed the approximately unbiased test of phylogenetic tree selection to assess if differences in topology between constrained and unconstrained gene trees are greater than expected by chance (Shimodaira 2002).

\section{Analysis of selective constraints}

The maximum likelihood (ML) approach of Yang et al. (2000), as implemented in the PAML package 4.0 (Yang 1997), was used to examine selection pressures acting on Veronicaceae $C Y C$-like genes. A likelihood ratio test (LRT) was used to test for positive selection (i.e., the 
presence of sites with $d_{\mathrm{N}} / d_{\mathrm{S}}$ rate ratio $\omega>1$ ). Positive selection is inferred if the LRTs show that models allowing for selection are significantly better than their null model. The level of significance is calculated as twice the $\log$ difference of the likelihood scores $(2 \Delta \ln L)$ estimated by each model. The null distribution of these results can be approximated by $\chi^{2}$ distribution with the number of degrees of freedom calculated as the difference in the number of estimated parameters between models. ML models that allow for heterogeneity in the $d_{\mathrm{N}} / d_{\mathrm{S}}$ ratio among lineages were also tested. Three models (one ratio model, free-ratio model, and two-ratio model) were used to determine if positive selection has acted along specific branches. Separate analyses were carried on specific internal branches of the phylogenetic tree (Fig. 2) to see if any branch has a $\omega$ that deviates significantly from the others.

\section{RNA in situ hybridization and RT-PCR}

RNA in situ hybridization was carried out essentially as described by Lincoln et al. (1994). A 950 bp PlCYC fragment was cloned into the vector pGEM3Z, the insert was PCR amplified using M13 universal forward and reverse primers to generate template for RNA probe preparation. Digoxigenin-labeled sense and antisense $P l C Y C$ riboprobes were generated by transcription from the SP6 and T7 promoters, respectively, and using an RNA labeling mix containing both Dig-labeled and non-labeled dUTP (50:50 molar ratio). Tissue for RT-PCR was collected from petals and stamens from mid-stage flowers (stigmas were emerged, sepals were longer than the petals, petals were longer than stamens, MPS); mid/late-stage flowers (petals were longer than sepals, stamens were elongated but not exserted, MLPS); and late-stage flowers (petals were enlarged and folded back, stamens were exserted, LPS), and from the inflorescence stem prior to stamen exsertion (flowers and pedicels removed, EIS), from the inflorescence stem after stamen exsertion (flowers and pedicels removed, LIS), and from the scape (SCA). Total RNA was isolated using an RNeasy Plant Mini Kit (Qiagen, Valencia, CA). Contaminating DNA was removed from RNA solutions using RNase-Free DNase (Qiagen, Valencia, CA) prior to RNA cleanup. The cDNA synthesis was carried out using $1 \mu \mathrm{g}$ of total RNA using a Transcriptor High Fidelity cDNA Synthesis Kit (Roche) and oligo dT primer. RT-PCR was carried out using PlCYC primers (CYCF, 5'-CATTGCGAGGAAGTTCTTCG-3'; CYCR, 5'-ACCCTTTACATCTGCTGGTC-3'). Fructose 1-6 bisphosphate aldolase 1 (Aldp1) was used as a positive control (ALDP1-F, 5'-GGACGAGCTCTCCAGCAG-3'; ALDP1-R, 5'-TGCAACCAAGCAAAGCTG-3'). RT-PCR was carried out using a LightCycler ${ }^{\circledR} 480$ System (Roche Applied Science) and LightCycler ${ }^{\circledR} 480$ SYBR Green I
Master mix. PCR conditions were $95^{\circ} \mathrm{C} 10$ min followed by $95^{\circ} \mathrm{C} 10 \mathrm{~s}, 58^{\circ} \mathrm{C} 10 \mathrm{~s}, 72^{\circ} \mathrm{C} 10 \mathrm{~s}$. PCR was terminated at the end of the linear phase, which was after 40 cycles for each set of reactions, the PCR products were run out on a $1 \%$ agarose gel and stained with $\mathrm{EtBr}$ for visualization.

Acknowledgments We thank Gwyneth Ingram, Justin Goodrich and Andrew Hudson for all their help with the in situ hybridizations, William Thompson for help with the initial amino acid alignment, Benjamin Pommerrenig for recommending the Fructose 1-6 bisphosphate aldolase primers for RT-PCR, Andrew Hudson, Dick Olmstead and Brenda Malloy for critical comments on the manuscript, Ica Dix for help with photography, and David Rawlinson for artwork. We acknowledge the SFI/HEA Irish Centre for High-End Computing (ICHEC) for the provision of computational facilities and support. This work was funded by a Basic Science Research Grant from Enterprise Ireland (JN).

\section{References}

Bello MA, Rudall PJ, Gonzalez F, Fernandez-Alonso JL (2004) Floral morphology and development in Aragoa (Plantaginaceae) and related members of the order Lamiales. Int J Plant Sci 16:723738

Bertin RI, Newman CM (1993) Dichogamy in angiosperms. Bot Rev 59:112-159

Bremer B, Bremer K, Heidari N, Erixon P, Olmstead RG, Anderberg AA, Kallersjo M, Barkhordarian E (2002) Phylogenetics of asterids based on 3 coding and 3 non-coding chloroplast DNA markers and the utility of non-coding DNA at higher taxonomic levels. Mol Phylogen Evol 24:274-301

Busch A, Zachgo S (2007) Control of corolla monosymmetry in the Brassicaceae Iberis amara. Proc Natl Acad Sci 104:1671416719

Carpenter R, Coen E (1990) Floral homeotic mutations produced by transposon-mutagenesis in Antirrhinum majus. Genes Dev 4:1483-1493

Citerne HL, Pennington RT, Cronk QC (2006) An apparent reversal in floral symmetry in the legume Cadia is a homeotic transformation. Proc Natl Acad Sci 103:12017-12020

Clark JI, Coen E (2002) The cycloidea gene can respond to a common dorsoventral prepattern in Antirrhinum. Plant J 30:639-648

Corley SB, Carpenter R, Copsey L, Coen E (2005) Floral asymmetry involves an interplay between TCP and MYB transcription factors in Antirrhinum. Proc Natl Acad Sci 102:5068-5073

Cubas P (2004) Floral zygomorphy, the recurring evolution of a successful trait. BioEssays 26:1175-1184

Cubas P, Lauter N, Doebley J, Coen E (1999a) The TCP domain: a motif found in proteins regulating plant growth and development. Plant J 18:215-222

Cubas P, Vincent C, Coen E (1999b) An epigenetic mutation responsible for natural variation in floral symmetry. Nature 401:157-161

Cubas P, Coen E, Zapater JMM (2001) Ancient asymmetries in the evolution of flowers. Curr Biol 11:1050-1052

Culley TM, Weller SG, Sakai AK (2002) The evolution of wind pollination in angiosperms. Trends in Ecol \& Evol 17:361-369

Doebley J, Stec A, Hubbard L (1997) The evolution of apical dominance in Maize. Nature 386:485-488

Donoghue MJ, Ree RH, Baum DA (1998) Phylogeny and the evolution of flower symmetry in the Asteridae. Trends Plant Sci $3: 311-317$ 
Endress PK (1997) Antirrhinum and Asteridae-evolutionary changes of floral symmetry. Symp Ser Soc Exp Biol 53:133-140

Endress PK (2001) Evolution of floral symmetry. Curr Opin Plant Biol 4:86-91

Feng X, Zhao Z, Tian Z, Xu S, Luo Y, Cai Z, Wang Y, Yang J, Wang Z, Weng L, Chen J, Zheng L, Guo X, Luo J, Sato S, Tabata S, Ma W, Cao X, Hu X, Sun C, Luo D (2006) Control of petal shape and floral zygomorphy in Lotus japonicus. Proc Natl Acad Sci 103:4970-4975

Friedman J, Barrett SCH (2008) A phylogenetic analysis of the evolution of wind pollination in the angiosperms. Intl J Plant Sci 169:49-58

Gaudin V, Lunness PA, Fobert PR, Towers M, Riou-Khamlichi C, Murray JA, Coen E, Doonan JH (2000) The expression of D-cyclin genes defines distinct developmental zones in snapdragon apical meristems and is locally regulated by the Cycloidea gene. Plant Physiol 122:1137-1148

Guindon S, Gascuel O (2003) A simple, fast, and accurate algorithm to estimate large phylogenies by maximum likelihood. Syst Biol 52:696-704

Henderson LB (1926) Floral anatomy of several species of Plantago. Am J Bot 13:397-406

Hileman LC, Baum DA (2003) Why do paralogs persist? Molecular evolution of CYCLOIDEA and related floral symmetry genes in Antirrhinae (Veronicaceae). Mol Biol Evol 20:591-600

Hileman LC, Kramar EM, Baum DA (2003) Differential regulation of symmetry genes and the evolution of floral morphologies. Proc Natl Acad Sci 100:12814-12819

Hubbard L, McSteen P, Doebley J, Hake S (2002) Expression patterns and mutant phenotype of teosinte branched 1 correlate with growth suppression in maize and teosinte. Genetics 162:1927-1935

Kalisz S, Ree RH, Sargent RD (2006) Linking floral symmetry genes to breeding system evolution. Trends Plant Sci 11:568-573

Katoh K, Kuma K, Toh H, Miyata T (2005) MAFFT version 5: improvement in accuracy of multiple sequence alignment. Nucleic Acids Res 33:511-518

Keane TM, Creevey CJ, Pentony MM, Naughton TJ, McInerney JO (2006) Assessment of methods for amino acid matrix selection and their use on empirical data shows that ad hoc assumptions for choice of matrix are not justified. BMC Evol Biol 6:29

Lartillot N, Brinkmann H, Philippe H (2007) Suppression of longbranch attraction artefacts in the animal phylogeny using a siteheterogeneous model. BMC Evol Biol 7(Suppl 1):S4

Lewis JM, Mackintosh CA, Shin S, Gilding E, Kravchenko S, Baldridge G, Zeyen R, Muehlbauer GJ (2008) Overexpression of the maize Teosinte Branchedl gene in wheat suppresses tiller development. Plant Cell Rep 27:1217-1225
Lincoln C, Long J, Yamaguchi J, Serikawa K, Hake S (1994) A knottedl-like homeobox gene in Arabidopsis is expressed in the vegetative meristem and dramatically alters leaf morphology when overexpressed in transgenic plants. Plant Cell 6:18591876

Luo D, Carpenter R, Vincent C, Copsey L, Coen E (1996) Origin of floral asymmetry in Antirrhinum. Nature 383:794-799

Luo D, Carpenter R, Copsey L, Vincent C, Clark J, Coen E (1999) Control of organ asymmetry in flowers of Antirrhinum. Cell 99:367-376

Notredame C, Higgins DG, Heringa J (2000) T-Coffee: A novel method for fast and accurate multiple sequence alignment. J Mol Biol 302:205-217

Olmstead RG, Jansen RK, Kim K-J, Wagstaff SJ (2000) The phylogeny of the Asteridae s.l. based on chloroplast $n d h \mathrm{~F}$ sequences. Mol Phylogenet Evol 16:96-112

Olmstead RG, dePamphilis CW, Wolfe AD, Young ND, Elisons WJ, Reeves PA (2001) Disintegration of the Scrophulariaceae. Am J Bot 88:348-361

Reeves PA, Olmstead RG (2003) Evolution of the TCP gene family in Asteridae: Cladistic and network approaches to understanding regulatory gene family diversification and its impact on morphological evolution. Mol Biol Evol 20:1997-2009

Shimodaira H (2002) An approximately unbiased test of phylogenetic tree selection. Syst Biol 51:492-508

Stebbins GL (1974) Flowering plants: evolution above the species level. Harvard University Press, USA

Swofford DL (1998) PAUP*: phylogenetic analysis using parsimony (*and other methods). Sinquer Sunderland, UK

Vieira C, Vieira J, Charlesworth D (1999) Evolution of the Cycloidea gene family in Antirrhinum and Misopates. Mol Biol Evol 16:1474-1483

Wang Z, Luo Y, Li X, Wang L, Xu S, Yang J, Weng L, Sato S, Tabata S, Ambrose M, Rameau C, Feng X, Hu X, Luo D (2008) Genetic control of floral zygomorphy in pea (Pisum sativum L.). Proc Natl Acad Sci 105:10414-10419

Weller SG, Sakai AK, Culley TM, Campbell DR, Dunbar-Wallis AK (2006) Predicting the pathway to wind pollination: heritabilities and genetic correlations of inflorescence traits associated with wind pollination in Schiedea salicaria (Caryophyllaceae). Jour Evol Bio 19:331-342

Yang Z (1997) PAML: a program package for phylogenetic analysis by maximum likelihood computer applications in the biosciences. Cabios 13:555-556

Yang Z, Nielsen R, Goldman N, Pedersen AM (2000) Codonsubstitution models for heterogeneous selection pressure at amino acid sites. Genetics 155:431-449 